Research Square

\title{
Impact of Serum Albumin Concentration and Neutrophil-lymphocyte Ratio Score on Gastric Cancer Prognosis
}

\author{
T. Costa \\ FMUP: Universidade do Porto Faculdade de Medicina \\ J. Nogueiro \\ CHUSJ: Centro Hospitalar Universitario de Sao Joao \\ D. Ribeiro \\ FMUP: Universidade do Porto Faculdade de Medicina \\ P. Viegas \\ FMUP: Universidade do Porto Faculdade de Medicina \\ H. Santos-Sousa ( $\square$ h.santos.sousa@gmail.com ) \\ CHUSJ: Centro Hospitalar Universitario de Sao Joao
}

\section{Research Article}

Keywords: Serum albumin concentration, neutrophil-to-lymphocyte ratio, gastric cancer prognosis, curative-intent resectional surgery

Posted Date: August 26th, 2021

DOI: https://doi.org/10.21203/rs.3.rs-814875/v1

License: (c) (i) This work is licensed under a Creative Commons Attribution 4.0 International License. Read Full License 


\section{Abstract}

Introduction/Aim

Serum albumin concentration (COA) and neutrophil-lymphocyte ratio (NLR) could reflect immunological and nutritional status. We aim to evaluate the impact of COA-NLR score on the prognosis of gastric cancer (GC).

Material and methods

We perform a retrospective analysis on a database of 637 GC cases, between January 2010 and December 2017. In 396 patients the inclusion criteria for this study were met (non-resectional or palliative surgery were excluded). Analytic data was only available in 203 patients. COA-NLR score was defined as: COA under $35 \mathrm{~g} / \mathrm{L}$ and NLR value of 2.585 or higher - score 2; one of these conditions - score 1 ; and neither - score 0.

Results

In our population ( $\mathrm{n}=203), 87$ patients were classified as score 0,82 as score 1 and 34 as score 2 . COA-NLR score was significantly associated with DFS [HR 1,674; CI95\% 1,115 - 2,513; $p=0,013$ ) and with OS [HR 2,072; CI95\% 1,531 - 2,805; p<0,001]. Kaplan-Meier curves analysis (log-rank test) revealed that a higher score of COA-NLR predicted a worse OS $(p<0,001)$ and DFS $(p=0,03)$. COA-NLR was an independent prognostic factor for OS when adjusted to $p S t a g e$ and age [adjusted HR 1,566; CI95\% 1,145 - 2,143; $p=0,005$ ].

Conclusions

Preoperative COA-NLR score was significantly associated with worse OS and DFS and, in this way, with worse prognosis on GC patients submitted to curative-intent resectional surgery.

\section{Introduction}

Gastric cancer (GC) is the fifth most common malignancy and the third leading cause of cancer-related death worldwide, according to GLOBOCAN $2018(1)$.

Surgical resection alongside with an appropriate lymphadenectomy remains the most effective therapy for gastric cancer. Despite the improvement of surgical techniques and medical therapies, GC prognosis remains unfavourable (2). Therefore, predictors of GC prognosis could support a better multidisciplinary clinical decision, founded in a more tailored perioperative management, treatment and follow-up strategies (2-5).

Diverse predictors of prognosis based on tumour behaviour, surgery approach and host-related features are being acknowledged (6-10). Regarding host-related features, patients immunological, nutritional status and systemic inflammatory response (SIR) have been associated with cancer prognosis. NLR is an easy, routinely and inexpensive marker of SIR that has been reported as a predictor of prognosis, in various clinical situations, namely on malignancy disorders (11-13). Commonly cancer patients are malnourished, either due to SIR and catabolic status that can induce muscle wasting and weight loss, or due to gastrointestinal tumour symptoms, as food intake impairment (14-16). Serum albumin concentration (COA) reflects nutritional status and SIR (17-19). Regarding gastric cancer, lower COA was associated with worse GC prognosis (20-23) and an recent meta-analysis showed an association between higher NLR and worse GC patients prognosis (13).

Sun et a/ hypothesized and evidenced the COA-NLR score as an predictor of worse postoperative survival after curative-intent surgery (24). Concerning the simplicity and reproductible applicability of this score to evaluate preoperative risk of poor GC patient's prognosis, our aim for this study is evaluate the impact of COA-NLR score on the prognosis of GC patients, submitted to curative-intent resectional surgery in our center.

\section{Material And Methods}

\subsection{Study design and population}

We performed a retrospective analysis on a prospective database of GC patients $(n=637)$, submitted to surgical treatment in an Upper GI Surgery Unit Tertiary Medical Center, between January 2010 and December 2017. The Institutional Review Board approved this study (CES 298-12).

In this study, only patients with gastric adenocarcinoma who were submitted to curative-intent surgery were included. The exclusion criteria were: palliative, prophylactic and completion gastrectomy (gastric stump cancer cases), non-resectional surgery, atypical resections, post endoscopic resections, pathological stage (pStage) IV, histologic types other than adenocarcinoma and R2 resections. Patients lost to follow-up were not included in this analysis. According to this selection criteria, a total of 396 cases were included and 203 were analysed (patients with analytical data available) (figure 1).

\subsection{Data Collection}


Demographic characterization of our population was based on the following parameters: age at time of surgery, gender, presence of comorbidities, American Society of Anaesthesiologists (ASA), physical status classification and body mass index (BMI) score.

Therapy approach characterization was based on the type of surgical resection and lymphadenectomy; quantification of lymph nodes retrieved; presence of conversion; presence of neoadjuvant therapy and resection classification (R). Resection was defined as curative (R0) when all gross disease was removed with negative margins, as incomplete when verified presence of positive surgical margins (R1) and when verified residual gross disease (R2).

For clinicopathological profile description, several parameters were evaluated, as the tumour location, size (cm) and type, macroscopic type, histologic type (Laurén classification) and growth pattern (Ming classification), TNM staging (7 ${ }^{\text {th }}$ edition, 2010), serum C-reactive protein (CRP) and lymphatic, venous and perineural invasion.

COA-NLR score analysis was based on pre-operative blood analysis, obtained until three weeks before the surgery, with data of serum concentration of albumin $(\mathrm{g} / \mathrm{L})$ and absolute count of neutrophils and lymphocyte $\left(10^{9} / \mathrm{L}\right)$ to calculate NLR (neutrophils absolute count / lymphocyte absolute count ). COA-NLR score was calculated based in our optimal cut-off values for NLR and on the standard cut-off for COA (35 $\mathrm{g} / \mathrm{L})$, as previously described (20). The optimal cut-off value of NLR $(2,585)$ for predicting overall survival (OS) (sensitivity 0,597 and specificity 0,574 ) was calculated based on the ROC curve (AUC 0,594, CI95\% 0,534-0,654, p=0,002) and Youden method (figure 2).

On that account, COA-NLR score was categorized as score COA-NLR 2 when COA under $35 \mathrm{~g} / \mathrm{L}$ and NLR value equal or higher than 2.585 , score COA-NLR 1 when one of these conditions was present (COA under $35 \mathrm{~g} / \mathrm{L}$ or NLR value equal or higher than 2.585), and score COA-NLR 0 when neither of these conditions were present (COA equal or higher that $35 \mathrm{~g} / \mathrm{L}$ and NLR lower 2.585) (figure 3).

Survival analysis assessed overall survival (OS) and disease-free survival (DFS). OS was defined as the period between surgery date and patient death date. For patients who had survived until the end of the observation period, their last follow-up visit data was considered for OS analysis. DFS was considered the period between the surgery and the first evidence of disease recurrence. Disease recurrence was based on clinical, radiologic or endoscopic indicators of the disease.

Median (range) follow-up was 37 (0-113) months and last follow-up review, for the entire study population, was in September of 2019.

\subsection{Perioperative management and surgical procedure}

For the diagnosis of GC, all patients underwent upper gastrointestinal endoscopy alongside biopsy and computerized tomography (CT). Tumour staging was established according to clinical, radiological (CT) or endoscopic (endoscopic ultrasonography) features and staging laparoscopy when considered necessary (mostly in locally advanced tumours with uncertain resectability).

Preoperative clinical stage of the patient was important to determine the surgical approach and the extent of lymphadenectomy. For diffuse and proximally located tumours, the surgery approach performed was a total gastrectomy with Roux-en-Y reconstruction. Additionally, for distally located tumours, the surgery approach performed was a subtotal distal gastrectomy, using Billroth II and sporadically Roux-en-Y reconstruction, according to the patient age and comorbidities. The extent of lymphadenectomy, according to the type of gastrectomy indicated, was classified based on the third version of Japanese Gastric Treatment Guidelines from 2010. Namely, D1 or D1+ was preferably performed in cT1N0 tumours and D2 in cN+ or cT2-T4 tumours (25).

After surgical intervention, all patients were admitted on a post-surgical intensive care unit for early extubation, pain control management, vigorous respiratory therapy, early mobilization and ambulation. Food intake was determined by post-operative clinical evolution. Patients were re-evaluated by the surgical team at 3-month intervals during the first year after surgery, every 6-months during the second year and annually thereafter.

\subsection{Statistical analysis}

Normal distribution of continuous variables was assessed by visual analysis of histograms, normal Q-Q plots and both Kolmogorov-Smirnov and Shapiro-Wilk test of normality. Non-parametric test Kruskal-Wallis was used to compare means and chi-square or Fisher's exact test were used to compare proportions, as appropriate.

ROC curves of NLR were plotted for OS and the optimal NLR cut-off value for predicting OS was determined using the Youden method.

Cumulative survival curves for OS and DFS were analysed by Kaplan-Meier (KM) method and adjusted to possible confounders, namely to pStage. Log rank test was performed to assess differences between COA-NLR score subgroups.

Univariate and multivariate analysis were performed to identify independent prognostic factors for OS and DFS. Variables significantly associated to OS and DFS in a univariate analysis were considered in a multivariate Cox proportional hazards model. 
Statistical analysis was performed using SPSS® 26.0 for Windows (IBM Co., Armonk, NY, USA). Significance was assumed for $p$ values inferior to 0.05 . All $p$ values given are results of 2 -sided tests.

\section{Results}

\subsection{Demographics and clinicopathological characteristics}

Of the patients included ( $\mathrm{n}=396), 203$ were eligible (completed analytical data) and had a mean (range) age of 66 (54-78) years and 59,6\% were males. Patients were categorized according to the COA-NLR score - $87(42,9 \%)$ to score $0,82(40,4 \%)$ to score 1 and 34 (16,7\%) to score 2 .

Population demographics and clinicopathological characteristics are described in table 1. COA-NLR score subgroups differed significantly on the mean age, ASA score, tumour size, pathological T stage, pathological stage, lymphatic permeation, perineural invasion, serum CRP concentration, surgery approach, type of lymphadenectomy and mean of lymph nodes retrieved (table 1).

Table 1 - Demographics and clinicopathological profile 


$$
\text { All COA-NLR Score }
$$

$\begin{array}{llll}(\mathrm{n}=203)(\%) & 0 & 1 & 2 \\ & (\mathrm{n}=87) & (\mathrm{n}=82) & (\mathrm{n}=34)\end{array}$

\begin{tabular}{llllll}
\hline $\begin{array}{l}\text { Demographics } \\
\text { Age at surgery (years), mean } \pm \text { SD }\end{array}$ & $66.1 \pm 12.5$ & $61.2 \pm 12.5$ & $68.01 \pm 11.6$ & $74.0 \pm 8.5$ & $<0.001^{1}$ \\
\hline Gender, $\mathbf{n}(\%)$ & & & & 0.134 \\
\hline Male & $121(59.6)$ & $45(51.7)$ & $53(64.6)$ & $23(67.6)$ & \\
\hline Female & $82(40.4)$ & $42(48.3)$ & $29(35.4)$ & $11(32.4)$ & \\
\hline Comorbidities, $\mathbf{n}(\%)$ & & & & & 0.409 \\
\hline Presence & $166(81.8)$ & $68(78.2)$ & $68(82.9)$ & $30(88.2)$ & \\
\hline Absence & $37(18.2)$ & $19(21.8)$ & $14(17.1)$ & $4(11.8)$ & \\
\hline ASA score, $\mathbf{n}(\%)$ & & & & 0.040 \\
\hline I & $29(14.3)$ & $14(16.1)$ & $11(13.4)$ & $4(11.8)$ & \\
\hline II & $110(54.2)$ & $50(57.5)$ & $49(59.8)$ & $11(32.4)$ & \\
\hline III & $59(29.1)$ & $22(25.3)$ & $19(23.2)$ & $18(52.9)$ & \\
\hline IV & $5(2.5)$ & $1(1.1)$ & $3(3.7)$ & $1(2.9)$ & \\
\hline & $25.86 \pm 4.19$ & $26.09 \pm 4.28$ & $25.36 \pm 3.95$ & $24.84 \pm 5.64$ & 0.278 \\
\hline
\end{tabular}

\section{BMI $\left(\mathrm{kg} / \mathrm{m}^{2}\right)$ mean \pm SD}

Therapeutic Approach

Surgery approach, $n$ (\%)

0.009

Open

$113(55.7) \quad 43(49.4) \quad 43(52.4) \quad 27(79.4)$

Laparoscopic

$90(44.3)$

$44(50.6)$

$39(47.6) \quad 7(20.6)$

Type of resection, $n$ (\%)

Total Gastrectomy

$84(41.4) \quad 41(47.1) \quad 34(41.5) \quad 9(26.5)$

Distal Gastrectomy, $109(53.7) \quad 39(44.8)$ $46(56.1) \quad 24(70.6)$

Billroth II

Distal Gastrectomy,

$10(4.9)$

$7(8.1$

$2(2.4)$

$1(2.9)$

Roux-en-Y

Type of lymphadenectomy, $n$ (\%)

$<0.001$

\begin{tabular}{|c|c|c|c|c|c|}
\hline D1 & $55(27.1)$ & $13(14.9)$ & $24(29.3)$ & $18(52.9)$ & \\
\hline D1+ & $75(36.9)$ & $33(37.9)$ & $30(36.6)$ & $12(35.3)$ & \\
\hline D2 & $73(36.0)$ & $41(47.2)$ & $28(34.1)$ & $4(11.8)$ & \\
\hline \multicolumn{6}{|l|}{ Resection Classification n (\%) } \\
\hline Ro & $197(97.0)$ & $86(98.9)$ & $80(97.6)$ & $31(91.2)$ & 0.076 \\
\hline R1 & $6(3.0)$ & $1(1.1)$ & $2(2.4)$ & $3(8.8)$ & \\
\hline Neoadjuvant treatment, n (\%) & & & & & 0.342 \\
\hline Presence & $31(15.3)$ & $17(19.5)$ & $10(12.2)$ & $4(11.8)$ & \\
\hline Absence & $172(84.7)$ & $70(80.5)$ & $72(87.8)$ & $30(88.2)$ & \\
\hline
\end{tabular}




\begin{tabular}{|c|c|c|c|c|c|}
\hline Tumor size $(\mathrm{cm})$, mean \pm SD & $4.31 \pm 2.82$ & $3.84 \pm 2.20$ & $4.85 \pm 3.14$ & $5.85 \pm 2.91$ & $<0.001^{1}$ \\
\hline Tumor location, n (\%) & & & & & 0.798 \\
\hline Proximal third (fundus) & $2(1.0)$ & $1(1.2)$ & $1(1.2)$ & $0(0.0)$ & \\
\hline Middle third (body) & $62(30.7)$ & $24(27.9)$ & $29(35.4)$ & $9(26.5)$ & \\
\hline Distal third (antrum/pylorus) & $131(64.9)$ & $59(68.6)$ & $48(58.5)$ & $24(70.6)$ & \\
\hline Extensive & $7(3.5)$ & $2(2.3)$ & $4(4.9)$ & $1(2.9)$ & \\
\hline Macroscopic type, n (\%) & & & & & 0.133 \\
\hline Fungating & $32(17.0)$ & $10(12,5)$ & $15(19.7)$ & $7(21.9)$ & \\
\hline Ulcerated & $54(28.7)$ & $29(36.3)$ & $18(23.7)$ & $7(21.9)$ & \\
\hline Infiltrative & $22(11.7)$ & $13(16.3)$ & $7(9.2)$ & $2(6.3)$ & \\
\hline Ulcero-fungating & $21(11.2)$ & $5(6.3)$ & $13(17.1)$ & $3(9.4)$ & \\
\hline Ulcero-infiltrative & $59(31.4)$ & $23(28.7)$ & $23(30.3)$ & $13(40.6)$ & \\
\hline Histologic type (Lauren), n (\%) & & & & & 0.602 \\
\hline Intestinal & $88(45.1)$ & $34(41.5)$ & $37(46.8)$ & $17(50.0)$ & \\
\hline Diffuse & $27(13.8)$ & $14(17.1)$ & $11(13.9)$ & $2(5.9)$ & \\
\hline Unclassified of solid structure & $6(3.1)$ & $1(1.2)$ & $4(5.1)$ & $1(2.9)$ & \\
\hline Unclassified of mixed structure & $51(26.2)$ & $25(30.5)$ & $17(21.5)$ & $9(26.5)$ & \\
\hline Unclassified NOS & $23(11.8)$ & $8(9.8)$ & $10(12.7)$ & $5(14.7)$ & \\
\hline Growth pattern (Ming), n (\%) & & & & & 0.517 \\
\hline Expansive & $43(21.9)$ & $15(18.1)$ & $21(26.6)$ & $7(20.6)$ & \\
\hline Infiltrative & $138(70.4)$ & $61(73.5)$ & $51(64.6)$ & $26(76.5)$ & \\
\hline Unclassified & $15(7.7)$ & $7(8.4)$ & $7(8.9)$ & $1(2.9)$ & \\
\hline pT ( $7^{\text {th }}$ ed, 2010$), n(\%)$ & & & & & 0.003 \\
\hline pT1-2 & $106(53.3)$ & $50(59.5)$ & $47(58.0)$ & $9(26.5)$ & \\
\hline рT3-4 & $93(46.7)$ & $34(40.5)$ & $34(42.0)$ & $25(73.5)$ & \\
\hline $\mathrm{pN}\left(7^{\text {th }}\right.$ ed, 2010$), \mathrm{n}(\%)$ & & & & & 0.071 \\
\hline $\mathrm{pN}-$ & $108(53.2)$ & $49(56.3)$ & $47(57.3)$ & $12(35.3)$ & \\
\hline $\mathrm{pN}+$ & $95(46.8)$ & $38(43.7)$ & $35(42.7)$ & $22(64.7)$ & \\
\hline Lymph nodes retrieved, mean $\pm S D$ & $27.57 \pm 12.46$ & $30.72 \pm 12.51$ & $26.63 \pm 12.10$ & $21.76 \pm 10.98$ & $<0.001^{1}$ \\
\hline LN ratio, n (\%) & & & & & 0.105 \\
\hline$\leq 0.2$ & $159(78.3)$ & $70(80.5)$ & $67(81.7)$ & $22(64.7)$ & \\
\hline$>0.2$ & $44(21.7)$ & $17(38.6)$ & $15(18.3)$ & $12(27.3)$ & \\
\hline \multicolumn{6}{|l|}{ Pathological stage, n (\%) } \\
\hline & & & & & 0.027 \\
\hline I & $91(45.09)$ & $44(51.2)$ & $40(48.8)$ & $7(20.6)$ & \\
\hline II & $49(24.3)$ & $19(22.1)$ & $20(24.4)$ & $10(29.4)$ & \\
\hline III & $62(30.7)$ & $23(26.7)$ & $22(26.8)$ & $17(50.0)$ & \\
\hline Lymphatic permeation, n (\%) & & & & & 0.053 \\
\hline Presence & $103(51.8)$ & $42(48.8)$ & $37(46.8)$ & $24(70.6)$ & \\
\hline Absence & $96(48.2)$ & $44(51.2)$ & $42(53.2)$ & $10(29.4)$ & \\
\hline
\end{tabular}


Venous invasion, $\mathrm{n}(\%)$

$\begin{array}{llllll}\text { Presence } & 67(33.7) & 25(29.1) & 26(32.9) & 16(47.1) \\ \text { Absence } & 132(66.3) & 61(70.9) & 53(67.1) & 18(52.9) & 0.019 \\ \text { Perineural invasion, } \mathbf{n}(\%) & & & & & \\ \text { Presence } & 65(32.7) & 23(26.7) & 24(30.4) & 18(52.9) & \\ \text { Absence } & 134(67.3) & 63(73.3) & 55(69.6) & 16(47.1) & \\ \text { CRP (mg/L) mean } \pm \text { SD } & 15.01 \pm 31.99 & 7.44 \pm 25.46 & 9.67 \pm 16.30 & 48.87 \pm 66.55 & <0.001^{1}\end{array}$

SD, standard deviation; ASA, American Society of Anesthesiologists; BMI, body mass index; NOS, none other specification; LN, lymph nodes; CRP, C reactive protein. ${ }^{1}$ Kruskal-Wallis Test.

Significant $\mathrm{p}$ values $(<0,05)$ are highlighted in bold.

\subsection{Survival analysis}

Regarding Kaplan-Meier curves (figure $4 \mathrm{a}, 4 \mathrm{~b})$, our population presented a mean ( \pm SD) OS of $70,0( \pm 6,8)$ months and a mean DFS of $86,4( \pm 6,6)$ months. Mean OS was significantly shorted in patients with higher COA-NLR score (34,3 versus 73,3 versus 82,7 months on score 2,1 and 0 , respectively) (table 2). When adjusted to pathological stage, mean OS was still significantly shorter in patients with higher COA-NLR score (table 2). Furthermore, mean DFS was also significantly shorter in higher COA-NLR score patients (69,3 versus 83,9 versus 94,13 months for score 2,1 and 0 , respectively) (table 2 ).

Table 2 - Comparative analysis between COA-NLR score groups: Survival analysis - Kaplan-Meier.

\begin{tabular}{|c|c|c|c|c|c|}
\hline & \multirow[t]{2}{*}{ All $n=203$} & \multicolumn{3}{|c|}{ COA-NLR Score } & \multirow[t]{2}{*}{$p$ value* } \\
\hline & & $\begin{array}{l}0 \\
(n=87)\end{array}$ & $\begin{array}{l}1 \\
(n=82)\end{array}$ & $\begin{array}{l}2 \\
(n=34)\end{array}$ & \\
\hline OS (months), mean \pm SD & $70.0 \pm 6.8$ & $82.7 \pm 10.1$ & $73.3 \pm 9.8$ & $34.3 \pm 13.8$ & $<0.001$ \\
\hline \multicolumn{6}{|c|}{ OS (months), adjusted to pStage, mean \pm SD } \\
\hline 1 & $88.3 \pm 8.6$ & $92.7 \pm 9.2$ & $84.3 \pm 12.8$ & $60.8 \pm 33.5$ & 0.023 \\
\hline II & $57.1 \pm 12.3$ & $50.4 \pm 12.2$ & $66.1 \pm 18.2$ & $23.2 \pm 16.9$ & 0.003 \\
\hline III & $51.1 \pm 11.8$ & $54.8 \pm 18.4$ & $55.5 \pm 17.3$ & $29.4 \pm 18.4$ & 0.021 \\
\hline DFS (months), mean \pm SD & $86.4 \pm 6.6$ & $94.3 \pm 8.6$ & $83.9 \pm 10.1$ & $69.3 \pm 20.1$ & 0.030 \\
\hline
\end{tabular}

SD, standard deviation; OS, overall survival; DFS, disease free survival.

${ }^{*}$ Calculated by Log rank test.

Significant $p$ values $(<0,05)$ are highlighted in bold.

\subsection{Prognostic Factors for OS and DFS}

A univariate analysis (table 3) revealed that age, tumour size, open surgery, type of lymphadenectomy, advanced pathological stage, presence of lymphatic permeation, venous invasion and perineural invasion and higher score of COA-NLR were significantly associated with OS. A multivariate analysis (table 3) identified age, pathological stage, type of surgical approach and COA-NLR score 2 as independent prognostic factors for OS.

A univariate analysis (table 3) indicated that tumour size, open surgery, advanced pathological stage, presence of lymphatic permeation, venous invasion and perineural invasion and higher score of COA-NLR were significantly associated with DFS. However, a multivariate analysis (table 3 ) identifies only pathological stage as an independent prognostic factor for DFS.

Table 3 - Prognostic factors for OS and DFS, univariate and multivariate analysis (Cox regression). 
PROGNOSTIC FACTORS FOR OS

Univariate analysis

$\begin{array}{lll}\mathrm{HR} & \mathrm{Cl} & \mathrm{p} \\ & 95 \% & \text { value }\end{array}$

Age

$1.037 \quad 1.022-<0.001$

1.052

Tumor size

$1.130 \quad 1.084-\quad<0.001$

1.178
PROGNOSTIC FACTORS FOR DFS

Univariate analysis

Multivariate analysis

$\begin{array}{ll}\mathrm{Cl} & \mathrm{p} \\ 95 \% & \text { value }\end{array}$

HR

$\begin{array}{ll}\mathrm{Cl} & \mathrm{p} \\ 95 \% & \text { value }\end{array}$

$1.028 \quad 1.005-$

0.01

(

$\begin{array}{lll}1.203 \quad 1.142- & <0.001 \\ & 1.268\end{array}$

\section{Type of surgery}

approach

Laparoscopic

Open

1

$\begin{array}{lll}1.953 \quad \begin{array}{l}1.422- \\ 2.682\end{array} & <0.001\end{array}$

\begin{tabular}{llllll}
1 & \multicolumn{5}{c}{1} \\
1.686 & $\begin{array}{l}1.030- \\
2.755\end{array}$ & $\mathbf{0 . 0 3 8}$ & 1.539 & $\begin{array}{c}1.009- \\
2.349\end{array}$ & $\mathbf{0 . 0 4 6}$
\end{tabular}

2.755

Type of

lymphadenectomy

\begin{tabular}{|c|c|c|c|c|c|c|c|c|c|}
\hline D1 & 1 & & & 1 & & & 1 & & \\
\hline D1+ & 1.004 & $\begin{array}{l}0.703- \\
1.433\end{array}$ & 0.984 & 9.175 & $\begin{array}{l}3.718- \\
22.64\end{array}$ & $<0.001$ & 8.058 & $\begin{array}{l}2.623- \\
24.75\end{array}$ & $<0.001$ \\
\hline D2+ & 0.570 & $\begin{array}{l}0.381- \\
0.851\end{array}$ & 0.006 & 24.31 & $\begin{array}{l}10.46- \\
56.40\end{array}$ & $<0.001$ & 14.673 & $\begin{array}{l}5.136- \\
41.92\end{array}$ & <. 001 \\
\hline
\end{tabular}

Pathologic Stage

\begin{tabular}{|c|c|c|c|c|c|c|c|c|c|}
\hline I & 1 & & & 1 & & & 1 & & \\
\hline$\|$ & 2.540 & $\begin{array}{l}1.659- \\
3.890\end{array}$ & $<0.001$ & 2.382 & $\begin{array}{l}1.321- \\
4.294\end{array}$ & 0.004 & 1.289 & $\begin{array}{l}0.708- \\
2.163\end{array}$ & 0.338 \\
\hline III & 4.439 & $\begin{array}{l}3.043- \\
6.474\end{array}$ & $<0.001$ & 2.923 & $\begin{array}{l}1.675- \\
5.101\end{array}$ & $\langle .001$ & 0.408 & $\begin{array}{l}0.790- \\
0.453\end{array}$ & 0.408 \\
\hline
\end{tabular}

Lymphatic

permeation

\begin{tabular}{llllllll} 
Absence & 1 & \multicolumn{1}{l}{} & & & \\
Presence & 2.699 & $1.924-$ & $<0.001$ & 3.338 & $2.154-$ & $<0.001$
\end{tabular}

Venous invasion

$\begin{array}{llllllll}\text { Absence } & 1 & & & & & \\ \text { Presence } & 2.268 & 1.667- & <0.001 & 4.354 & 2.794- & <0.001\end{array}$

Perineural invasion

$\begin{array}{lllllll}\text { Absence } & 1 & & & 1 & & \\ \text { Presence } & 2.186 & 1.612- & <0.001 & 1.540 & 0.793- & 0.202 \\ & & 2.966 & & & 2.989 & \\ \text { COA-NLR } & & & 2.854 & 1.278- & \mathbf{0 . 0 1 1} \\ & & & 6.373 & \end{array}$

\begin{tabular}{lllllll} 
COA-NLR 0 & 1 & \multicolumn{5}{c}{1} \\
COA-NLR 1 & 1.355 & $0.800-$ & 0.259 & 1.209 & $0.706-$ & 0.489 \\
& & 2.296 & & & 2.068 & \\
COA-NLR 2 & 4.205 & $\begin{array}{c}2.417- \\
7.316\end{array}$ & $<0.001$ & 2.263 & $\begin{array}{c}1.251- \\
4.093\end{array}$ & $\mathbf{0 . 0 0 7}$
\end{tabular}

HR, hazard ratio; $\mathrm{Cl} 95 \%$, confidence interval 95\%; OS, overall survival. Significant $p$ values $(<0.05)$ are highlighted in bold.

\section{Discussion}


We intended to evaluate the impact of COA-NRL score on the prognosis of GC patients, submitted to curative-intent resectional surgery.

Gastric cancer is an inflammation-driven cancer where inflammatory responses act as an important role on tumour development and progression (26). Patient's outcomes depend on tumour-related factor, therapy approach features and, also, on host-related factors, such as patient's immunological and nutritional status and SIR (6-10).

Malnutrition is known to be prevalent among cancer patients and it is associated with higher risk of postoperative complications and poor prognosis. Nutrition status evaluation is an important step of cancer care management and treatment decisions (27). On a daily practice, the serum protein albumin has been used as a simple and reproducible parameter to assess nutrition status. Prior studies described serum albumin as an independent predictor of survival outcome in several types of cancer, such as on GC where lower COA was associated with worse GC patient's prognosis $(20-23,28)$. SIR, as an important role in the progressive nutritional and functional decline, has been displayed as a plausible confounding factor to the impact of serum albumin concentration on cancer patient's survival $(20,21,29)$. Considering this, measurement of SIR has been included in the definition of cancer cachexia alongside with weight loss and sarcopenia (30). It is widely accepted the relationship between inflammation and cancer development (31). NLR has being widely presented as an index of SIR and studied in diverse clinical disorders, such as critically ill and cancer patients $(9,11-13,32-36)$. NLR proposes to evaluated the inverse relationship between neutrophils and lymphocytes, based on the SIR and on cancer patient's immunological status. Recent studies have suggested that NLR could be used as a prognostic marker in malignance disorder, with a meta-analysis showing an association between higher NLR and worse GC patients' prognosis (13).

Host-related factors assessment, such as nutrition, inflammatory and immune status, is essential on management and treatment strategy decisions of cancer patients. Preoperative nutritional care on moderately or severely malnourished gastrointestinal cancer patients showed to reduce postoperative morbidity and mortality (37). Thereby, COA-NLR score, as a simple and reproducible parameter, could be used to facilitate a more practical assessment and then address a more appropriate and individualized therapy approach. Higher COA-NLR score aims to define GC patients with higher risk of poor prognosis, based on their malnutrition and inflammatory status. This score was first described by Sun et al. and was displayed as a predictor of OS, independent of TNM stage. In their study, COA-NLR score was significantly associated with poor prognosis and was able to classify GC patients into three independent groups, before curative-intent surgery (24).

In our study, patients with higher COA-NLR score were significantly characterized by higher age and higher ASA score, acknowledged predictors of poor tolerance to an illness status. Patients classified as COA-NLR 2 were also significantly associated with worse clinicopathological features such as higher tumour size, advanced pathological stage and with more lymphatic permeation and perineural invasions. Likewise, COA-NLR score 2 patients had significantly higher CRP concentration, an acute-phase protein also used to evaluate inflammatory status. In this way, our analysis showed a significantly association between higher COA-NLR score and poor prognosis features. . For our studied population, higher COA-NLR scores were significantly associated with lower OS and lower DFS. Concerning adjustment to pathological stage, survival analysis showed that independent of pathological stage higher COA-NLR scores were still significantly associated with lower OS. We performed a multivariate analysis that exposed age, type of surgery approach, pathological stage and COA-NLR score 2 as independent prognostic factors for OS and only pathological stage as an independent prognostic factor for DFS. In this manner, our findings support Sun et a/ findings and picture COA-NLR score as a conceivable and valuable predictor of OS in GC patients.

Multidisciplinary assessment of GC patients would benefit on easy and simple score capable of predict prognosis. A prospective study regarding the effect of preoperative nutrition on patients with GC showed a lower 3-year OS and DFS in malnourished patients than in those receiving adequate nutrition (38). Another study, besides showing that 3-year OS and DFS rates were significantly lower in malnourished GC patient, also showed that preoperative correction of hypoalbuminemia was significantly associated with higher OS and DFS, for pStage II and III (39). These results suggest that preoperative status in GC patients should be optimized. Despite the several prognostic predictors describe on the literature, COA-NLR might be a reproducible score able to identify patients at risk of poor prognosis after curative-intend surgery. And in this way, help the multidisciplinary team on the decision of the most appropriate and individualized therapy approach, through a personalised perioperative optimization of patient's status.

By these means, we believe that our study may help the multidisciplinary team on the decision of the most appropriate therapy approach. The limitations of the present study includes its retrospective nature; analysis of a limited number of patients $(n=396)$ belonging only to one hospital; the selected hospital is a tertiary center - receiving patients in poorer health conditions and may not be representative of the general population; only patients submitted to curative intent surgery were included in the study. Further internal and external validations are needed to clarify the accurate prognostic role of preoperative COA-NLR score.

\section{Declarations}

Funding - No funding.

Conflicts of interest/Competing interests - No conflicts of interest. 
Availability of data and material - Yes.

Code availability - Not applicable.

Authors' contributions - All authors contributed to the study conception and design. Material preparation, data collection and analysis were performed by TC, JN, DR, PV and HSS. The first draft of the manuscript was written by TC and all authors commented on previous versions of the manuscript. All authors read and approved the final manuscript.

Ethics approval - Ethics approval by ethics committee.

Consent to participate - Not applicable.

Consent for publication - Not applicable.

\section{References}

1. Bray F, Ferlay J, Soerjomataram I, Siegel RL, Torre LA, Jemal A. Global cancer statistics 2018: GLOBOCAN estimates of incidence and mortality worldwide for 36 cancers in 185 countries. CA: A Cancer Journal for Clinicians. 2018;68(6):394-424.

2. Sitarz R, Skierucha M, Mielko J, Offerhaus GJA, Maciejewski R, Polkowski WP. Gastric cancer: epidemiology, prevention, classification, and treatment. Cancer Manag Res. 2018;10:239-48.

3. Smyth EC, Verheij M, Allum W, Cunningham D, Cervantes A, Arnold D. Gastric cancer: ESMO Clinical Practice Guidelines for diagnosis, treatment and follow-up. Ann Oncol. 2016;27(suppl 5):v38-v49.

4. Ushijima T, Sasako M. Focus on gastric cancer. Cancer Cell. 2004;5(2):121-5.

5. Jankowski M, Las-Jankowska M, Sousak M, Zegarski W. Contemporary enteral and parenteral nutrition before surgery for gastrointestinal cancers: a literature review. World J Surg Oncol. 2018;16(1):94-

6. McMillan DC. Systemic inflammation, nutritional status and survival in patients with cancer. Current opinion in clinical nutrition and metabolic care. 2009;12(3):223-6.

7. Wang SY, Yeh CN, Lee HL, Liu YY, Chao TC, Hwang TL, et al. Clinical impact of positive surgical margin status on gastric cancer patients undergoing gastrectomy. Ann Surg Oncol. 2009;16(10):2738-43

8. Setala LP, Kosma VM, Marin S, Lipponen PK, Eskelinen MJ, Syrjanen KJ, et al. Prognostic factors in gastric cancer: the value of vascular invasion, mitotic rate and lymphoplasmacytic infiltration. Br J Cancer. 1996;74(5):766-72.

9. Roxburgh CS, McMillan DC. Role of systemic inflammatory response in predicting survival in patients with primary operable cancer. Future Oncol. 2010;6(1):149-63.

10. Mohri Y, Tanaka K, Ohi M, Yokoe T, Miki C, Kusunoki M. Prognostic significance of host- and tumor-related factors in patients with gastric cancer. World J Surg. 2010;34(2):285-90.

11. Kim JH, Han DS, Bang HY, Kim PS, Lee KY. Preoperative neutrophil-to-lymphocyte ratio is a prognostic factor for overall survival in patients with gastric cancer. Ann Surg Treat Res. 2015;89(2):81-6.

12. Kumar R, Geuna E, Michalarea V, Guardascione M, Naumann U, Lorente D, et al. The neutrophil-lymphocyte ratio and its utilisation for the management of cancer patients in early clinical trials. Br J Cancer. 2015;112(7):1157-65.

13. Chen J, Hong D, Zhai Y, Shen P. Meta-analysis of associations between neutrophil-to-lymphocyte ratio and prognosis of gastric cancer. World journal of surgical oncology. 2015;13:122.

14. Mantzorou M, Koutelidakis A, Theocharis S, Giaginis C. Clinical Value of Nutritional Status in Cancer: What is its Impact and how it Affects Disease Progression and Prognosis? Nutrition and cancer. 2017;69(8):1151-76.

15. Ravasco P. Nutrition in Cancer Patients. Journal of clinical medicine. 2019;8(8).

16. Santarpia L, Contaldo F, Pasanisi F. Nutritional screening and early treatment of malnutrition in cancer patients. J Cachexia Sarcopenia Muscle. 2011;2(1):27-35. 
17. Feinberg J, Nielsen EE, Korang SK, Halberg Engell K, Nielsen MS, Zhang K, et al. Nutrition support in hospitalised adults at nutritional risk. Cochrane Database Syst Rev. 2017;5(5):CD011598-CD.

18. Yang J-R, Xu J-Y, Chen G-C, Yu N, Yang J, Zeng D-X, et al. Post-diagnostic C-reactive protein and albumin predict survival in Chinese patients with non-small cell lung cancer: a prospective cohort study. Scientific Reports. 2019;9(1):8143.

19. Song GM, Liu XL, Bian W, Wu J, Deng YH, Zhang H, et al. Systematic review with network meta-analysis: comparative efficacy of different enteral immunonutrition formulas in patients underwent gastrectomy. Oncotarget. 2017;8(14):23376-88.

20. Crumley AB, Stuart RC, McKernan M, McMillan DC. Is hypoalbuminemia an independent prognostic factor in patients with gastric cancer? World journal of surgery. 2010;34(10):2393-8.

21. Onate-Ocana LF, Aiello-Crocifoglio V, Gallardo-Rincon D, Herrera-Goepfert R, Brom-Valladares R, Carrillo JF, et al. Serum albumin as a significant prognostic factor for patients with gastric carcinoma. Annals of surgical oncology. 2007;14(2):381-9.

22. Lien YC, Hsieh CC, Wu YC, Hsu HS, Hsu WH, Wang LS, et al. Preoperative serum albumin level is a prognostic indicator for adenocarcinoma of the gastric cardia. Journal of gastrointestinal surgery : official journal of the Society for Surgery of the Alimentary Tract. 2004;8(8):1041-8.

23. Toiyama $\mathrm{Y}$, Yasuda H, Ohi M, Yoshiyama S, Araki T, Tanaka K, et al. Clinical impact of preoperative albumin to globulin ratio in gastric cancer patients with curative intent. American journal of surgery. 2017;213(1):120-6.

24. Sun X, Wang J, Liu J, Chen S, Liu X. Albumin concentrations plus neutrophil lymphocyte ratios for predicting overall survival after curative resection for gastric cancer. OncoTargets and therapy. 2016;9:4661-9.

25. Japanese gastric cancer treatment guidelines 2010 (ver. 3). Gastric cancer : official journal of the International Gastric Cancer Association and the Japanese Gastric Cancer Association. 2011;14(2):113-23.

26. Hanahan D, Weinberg RA. Hallmarks of cancer: the next generation. Cell. 2011;144(5):646-74.

27. Benoist S, Brouquet A. Nutritional assessment and screening for malnutrition. Journal of visceral surgery. $2015 ; 152$ Suppl 1:S3-7.

28. Gupta D, Lis CG. Pretreatment serum albumin as a predictor of cancer survival: a systematic review of the epidemiological literature. Nutrition journal. 2010;9:69.

29. McMillan DC. An inflammation-based prognostic score and its role in the nutrition-based management of patients with cancer. The Proceedings of the Nutrition Society. 2008;67(3):257-62.

30. Vanhoutte G, van de Wiel M, Wouters K, Sels M, Bartolomeeussen L, De Keersmaecker S, et al. Cachexia in cancer: what is in the definition? BMJ open gastroenterology. 2016;3(1):e000097.

31. Greten FR, Grivennikov SI. Inflammation and Cancer: Triggers, Mechanisms, and Consequences. Immunity. 2019;51(1):27-41.

32. Hajibandeh S, Hajibandeh S, Hobbs N, Mansour M. Neutrophil-to-lymphocyte ratio predicts acute appendicitis and distinguishes between complicated and uncomplicated appendicitis: A systematic review and meta-analysis. American journal of surgery. 2020;219(1):154-63.

33. Jiang J, Liu R, Yu X, Yang R, Xu H, Mao Z, et al. The neutrophil-lymphocyte count ratio as a diagnostic marker for bacteraemia: A systematic review and meta-analysis. The American journal of emergency medicine. 2019;37(8):1482-9.

34. Hwang SY, Shin TG, Jo IJ, Jeon K, Suh GY, Lee TR, et al. Neutrophil-to-lymphocyte ratio as a prognostic marker in critically-ill septic patients. The American journal of emergency medicine. 2017;35(2):234-9.

35. Zahorec R. Ratio of neutrophil to lymphocyte counts-rapid and simple parameter of systemic inflammation and stress in critically ill. Bratislavske lekarske listy. 2001;102(1):5-14.

36. Templeton AJ, McNamara MG, Seruga B, Vera-Badillo FE, Aneja P, Ocana A, et al. Prognostic role of neutrophil-to-lymphocyte ratio in solid tumors: a systematic review and meta-analysis. J Natl Cancer Inst. 2014;106(6):dju124.

37. Wu GH, Liu ZH, Wu ZH, Wu ZG. Perioperative artificial nutrition in malnourished gastrointestinal cancer patients. World journal of gastroenterology. 2006;12(15):2441-4.

38. Yamagata Y, Yoshikawa T, Yura M, Otsuki S, Morita S, Katai H, et al. Current status of the "enhanced recovery after surgery" program in gastric cancer surgery. Annals of gastroenterological surgery. 2019;3(3):231-8.

Page $11 / 15$ 
Figures

\title{
INITIAL POPULATION (n=637)
}

\author{
January 2010 - December 2017
}

Patients treated in an Upper GI Surgery Unit

\section{EXCLUSION CRITERIA (N=229)}

55 - Non resectional surgery

48 - Palliative resection

51 - Pathological stage IV

25 - Histologic type other than adenocarcinoma

23 - Completion gastrectomy

13 - Prophylactic gastrectomy

8 - Atypical gastrectomy

4 - Post endoscopic resection

2 - $\mathrm{R} 2$ resection

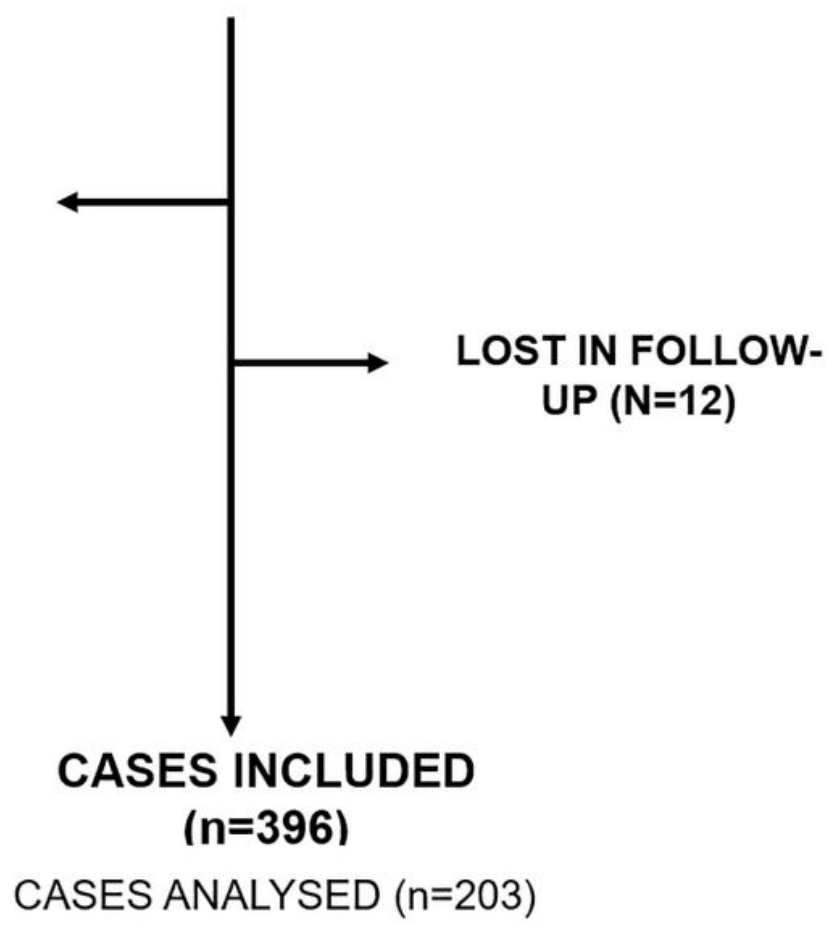

Figure 1

Flow Chart of the study design 


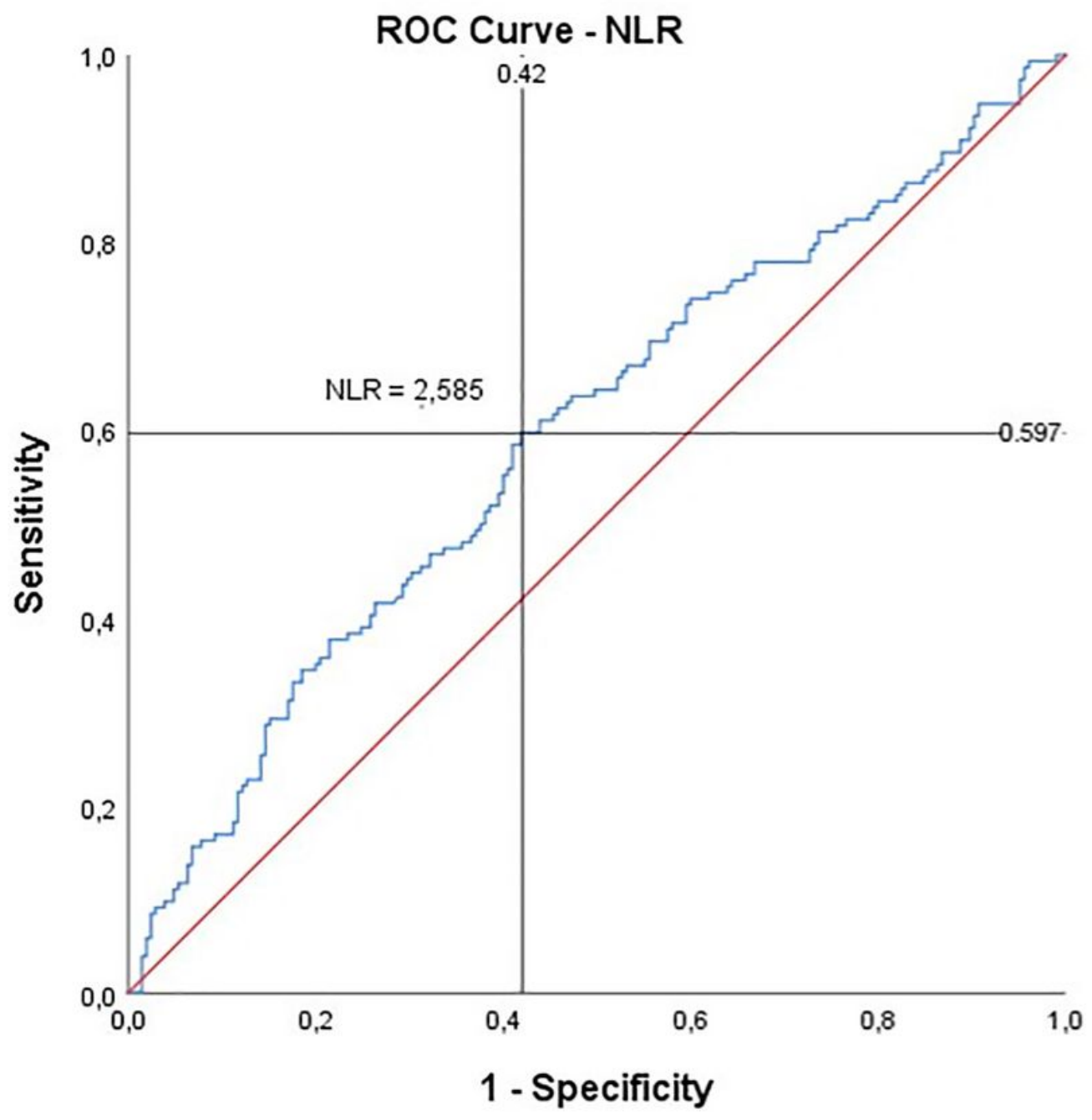

Figure 2

ROC curve for NLR. Optimal cut-off value of NLR (2.585) for predicting OS, based on Youden method. 


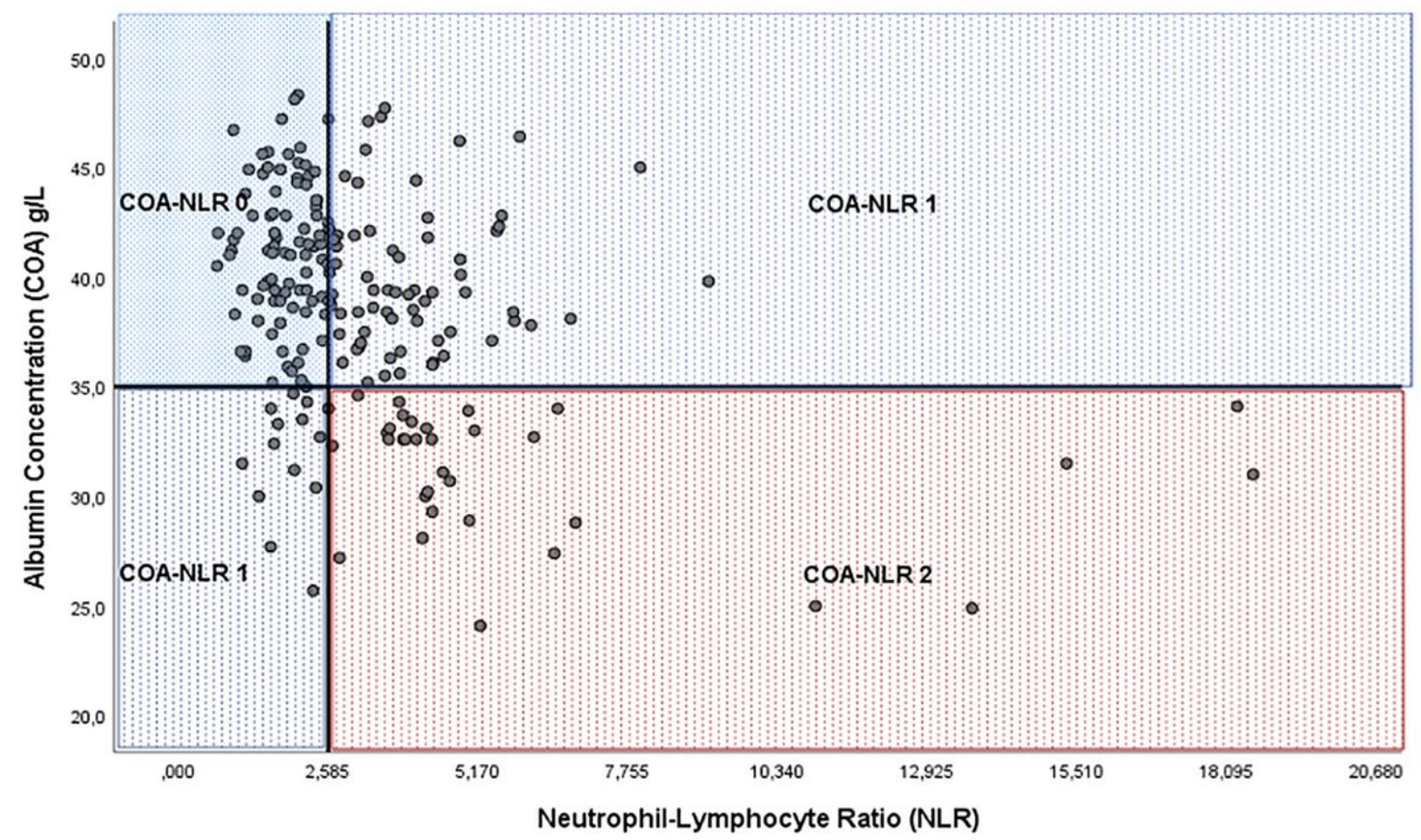

\begin{tabular}{lll}
\hline COA-NLR 0 & $\mathrm{COA} \geq 35 \mathrm{~g} / \mathrm{L}+\mathrm{NLR}<2,585$ & $\mathrm{n}=87$ \\
\hline COA-NLR 1 & $\mathrm{COA} \geq 35 \mathrm{~g} / \mathrm{L}+\mathrm{NLR} \geq 2,585$ & $\mathrm{n}=82$ \\
COA-NLR 2 & $\mathrm{COA}<35 \mathrm{~g} / \mathrm{L}+\mathrm{NLR}<2,585$ & \\
& $\mathrm{COA}<35 \mathrm{~g} / \mathrm{L}+\mathrm{NLR} \geq 2,585$ & $\mathrm{n}=34$
\end{tabular}

Figure 3

The fundamentals and distribution of COA-NLR score. COA-NLR score $0(n=87)$, COA-NLR score $1(n=82)$ and COA-NLR score 2 ( $n=34)$. 


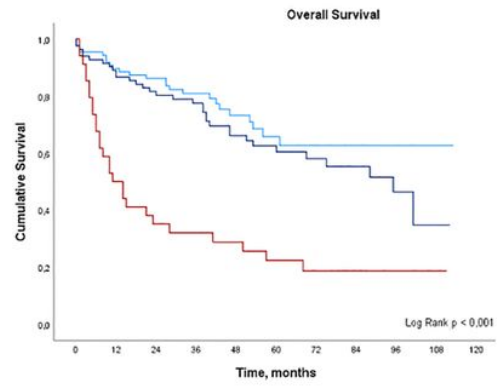

$4 a$

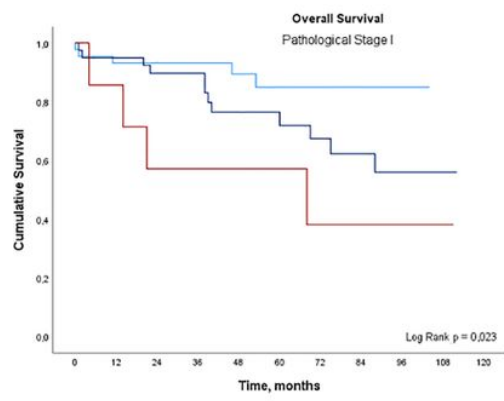

$4 c$

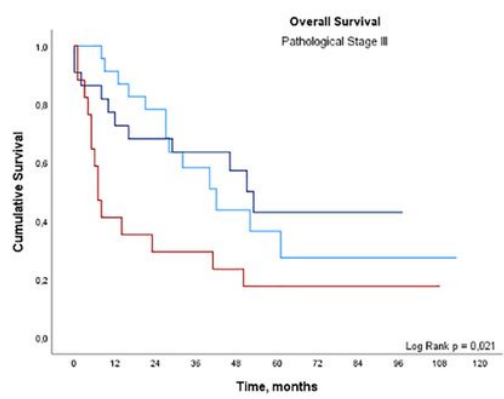

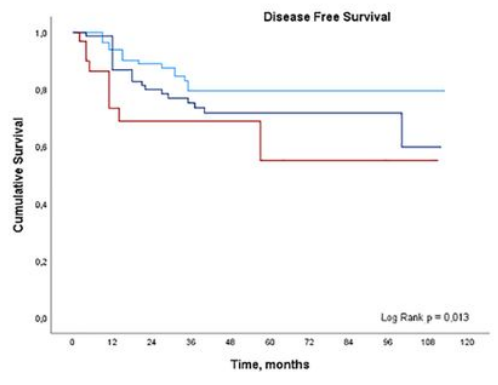

$4 b$

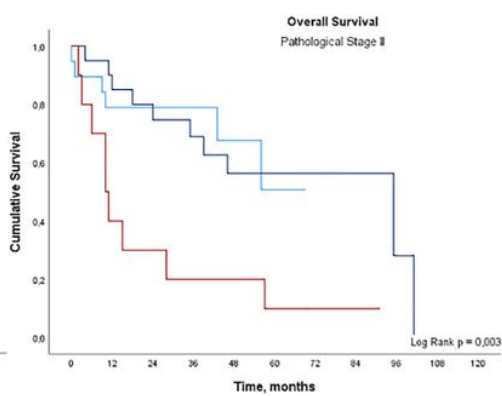

4d

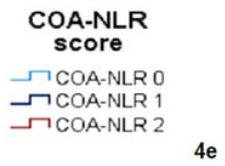

Figure 4

Survival analysis, with Kaplan-Meier curves for OS (4a) and DFS (4b). Kaplan-Meier curves for OS, adjusted do pStage I (4c), II (4d) and III (4e). KM, Kaplan-Meier; OS, Overall survival; DFS, disease free survival; pStage, pathological stage 